\title{
Enhancing the Stability of the Switched Systems Using the Saltation Matrix
}

\author{
Juan-Guillermo Muñoz*, Arnold Pérez ${ }^{\dagger}$ and Fabiola Angulo \\ Departamento de Ingeniería Eléctrica, Electrónica y Computación \\ Percepción y Control Inteligente - Bloque Q, Campus La Nubia \\ Manizales 170003, Colombia \\ Facultad de Ingeniería y Arquitectura \\ Universidad Nacional de Colombia - Sede Manizales \\ *jgmunozc@unal.edu.co \\ †aperezco@unal.edu.co \\ \$fangulog@unal.edu.co
}

Received 9 March 2018

Accepted 17 November 2018

Published 4 January 2019

\begin{abstract}
Controlling switched systems is a difficult task, even when dealing with piecewise linear systems (CPWLs), which consist of a set of linear differential equations and a set of switching conditions. This difficulty is largely due to the loss of linearity in the entire system, and it is necessary to solve differential and algebraic equations to determine the solution. In this paper, a new method to tune the parameters of the controllers applied to switched systems is derived using information from the saltation matrix, particularly its induced norm. First, the parameters are tuned using classical methods, and then, after analyzing the norm of the saltation matrix, a new set of parameters that guarantees the stability of the period-1 orbit is obtained. The method is validated using analytical solutions for two different systems (boost and boost-flyback power converters) and is also experimentally validated for the boost-flyback power converter.
\end{abstract}

Keywords: Monodromy matrix; saltation matrix; matrix norm; current-mode-control; power converters.

\section{Introduction}

A switched system is a system that changes its topology according to an event. For this reason, switched systems are defined by a set of ordinary differential equations and a set of switching conditions. Switched systems are present in chemical

\footnotetext{
†Corresponding author.
}

This is an Open Access article published by World Scientific Publishing Company. It is distributed under the terms of the Creative Commons Attribution 4.0 (CC-BY) License. Further distribution of this work is permitted, provided the original work is properly cited. 
processes, ${ }^{1}$ aeronautics, ${ }^{2}$ physics, ${ }^{3}$ communications,${ }^{4}$ automotive industry, ${ }^{5}$ renewable energies ${ }^{6,7}$ and power electronics, ${ }^{8,9}$ among many others. As electronic devices have gained an important place in modern life, the design of new and robust controllers plays a critical role in the development of these devices. Although the relation between stability and other mathematical concepts, such as the measure matrix, matrix norm, and so on, has been known for many years, ${ }^{10-12}$ its use has been minimal in the design and computation of controllers compared with other tools, such as PID, robust and adaptive controllers.

One of the most popular methods to analyze switched systems is to describe them as Filippov systems. ${ }^{13}$ If the system switches among linear topologies, it is called a piecewise linear system (PWL). If the system has a period-T orbit, it is possible to determine its stability through the Floquet exponents by using the Filippov theory, the information of the periodic orbit and the saltation matrix. ${ }^{14}$

Power converters are a type of switched system that can be used to analyze, compute and validate new controllers since their experimental set-up allows for the testing of analyses and results. Boost and boost-flyback converters allow for output voltages that are more than twice the input voltage. ${ }^{15}$ These converters can be used in different applications, such as fuel cells ${ }^{16}$ and photovoltaic sources. ${ }^{17}$ Boost converters have a simple topology and have been studied with different controllers. One of the most frequently used controller is the peak current mode control, which uses an outer voltage loop and an inner current. ${ }^{18}$ When the switch is on for a period of time greater than $50 \%$ of the period $T$, the converters tend to subharmonic or chaotic solutions. Therefore, it is necessary to attach a compensation ramp to avoid these types of solutions. ${ }^{19,20}$ Although this compensation ramp adds stability to the system, as the slope increases, the response tends to be slower.

A simple control strategy using the saltation matrix applied to a buck power converter is described in Ref. 21; in this study, a high frequency and low amplitude signal is added to the compensation ramp to enhance the stability range of the period- 1 orbit. In the same paper, a boost power converter is analyzed and controlled, thereby increasing the value of the slope of the compensation ramp. However, as this value increases, the response of the system deteriorates, mainly when light loads are present. ${ }^{22}$ In Ref. 23 , a controller is proposed that forces the system to have more than two switchings during a period in an attempt to avoid subharmonic behaviors in complex converters; however, as the switching time increases, faster devices are needed and the durability of the elements decreases. Other studies that include both fast scale and slow scale instabilities are reported in Refs. 24 and 25; these instabilities appear due to the use of large parameters or high frequency phenomena.

Taking into account previous works and the possibilities provided by the matrix norm concept, it proposes modifying the saltation matrix modifying the switching function by changing the controllers. This change is achieved using the matrix norm and computing the new values of the controllers so that a change in the norm of the 
saltation matrix is obtained and the stability of the period-1 orbit is guaranteed over a wider range. The main contribution of this paper is to extend the stability range of the period-1 orbit in switched systems by re-tuning the initial parameters of the controller and employing the matrix norm of the saltation matrix as the objective of control as opposed to using a new controller. Tuning is carried out offline.

The paper is organized as follows: in Sec. 2, mathematical concepts, such as the monodromy matrix, saltation matrix and matrix norm are introduced. In Sec. 3, the stability of the period-1 orbit in a boost and boost-flyback converter is analyzed using the monodromy matrix. In Sec. 4, the method to re-tune the control parameters and the analytical results are presented. In Sec. 5, an experimental set-up is used to validate the proposed approach. Finally, in Sec. 6, the main conclusions are shown.

\section{Mathematical Preliminaries}

This section introduces two important concepts of this paper: the saltation matrix and the matrix norm induced by the vector norm.

\subsection{Monodromy and saltation matrices}

The monodromy matrix is a powerful tool that is used to determine the stability of a periodic orbit in discontinuous systems. ${ }^{14,26}$ The monodromy matrix is the fundamental matrix of a system of ODEs evaluated at the period $T$ of the coefficients of the system. The evolution of a period- 1 orbit during a period $T$ of a system with two topologies is schematically depicted in Fig. 1(a). The system starts at $t=0$ and evolves according to the flux $\phi_{1}$ in the subspace $V_{-}$. When an event occurs at $t=t_{p}$, the system changes its topology and evolves with the flux $\phi_{2}$ until $t=T$, according to the dynamics defined in the subspace $V_{+}$. At $t=T$, the topology changes again

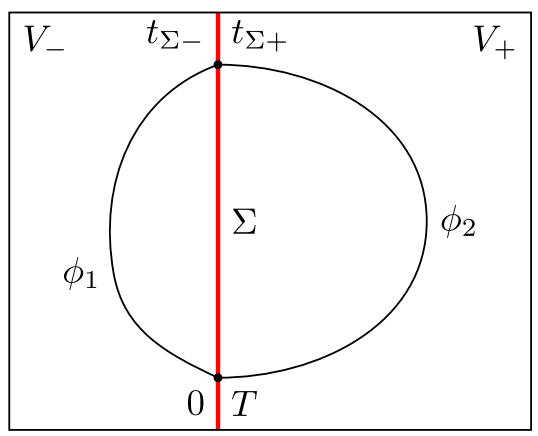

(a) Periodic orbit with switching surface

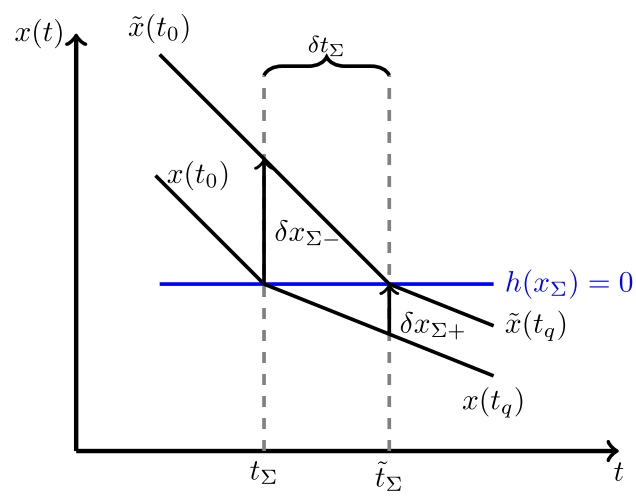

(b) Disturbed and undisturbed solution

Fig. 1. Typical periodic solution of a switched system. 
and the cycle is repeated. The condition to change topologies is given in a general form as $h(x(t))=0$ and is represented as the hypersurface $\Sigma$. A system with two topologies can be described using the Filyppov theory in the following way:

$$
\dot{X}(t) \in F(X(t))= \begin{cases}f_{-}(t, X(t)) & X \epsilon V_{-} \\ \overline{c o}\left\{f_{-}(t, X(t)), f_{+}(t, X(t))\right\} & X \epsilon \Sigma \\ f_{+}(t, X(t)) & X \epsilon V_{+}\end{cases}
$$

where the vector fields before and after the computation are $f_{-}$and $f_{+}$, respectively, and $\overline{c o}\left\{f_{-}, f_{+}\right\}$is the convex set between the vector fields, defined as

$$
\overline{c o}\left\{f_{-}, f_{+}\right\}=\left\{(1-q) f_{-}+q f_{+}, \forall q \in[0,1]\right\} .
$$

The period-1 solution of a piece-wise linear system with two topologies is

$$
\begin{aligned}
& x\left(t_{\Sigma}\right)=e^{A_{1}\left(t_{\Sigma}\right)} x(0)+\int_{0}^{t_{\Sigma}} e^{A_{1}\left(t_{\Sigma}-\tau\right)} B_{1} d \tau \\
& x(T)=e^{A_{2}\left(T-t_{\Sigma}\right)} x\left(t_{\Sigma}\right)+\int_{t_{\Sigma}}^{T} e^{A_{2}(T-\tau)} B_{2} d \tau,
\end{aligned}
$$

where $t_{\Sigma}$ is the switching instant. To analyze the stability of the period-1 orbit, it is necessary to know the value of the periodic orbit, the switching instants $\left(t_{\Sigma}\right)$ and the values of the corresponding state at these times $\left(x\left(t_{\Sigma}\right)\right)$. After these data are determined, the initial condition is adjusted (see Fig. 1(b)) and corrections in the monodromy matrix at the switching times are introduced from the saltation matrices. ${ }^{27}$ The monodromy matrix is given by

$$
M_{m}(T, 0)=\hat{\phi}_{2} S \hat{\phi}_{1},
$$

where the solution matrices are $\hat{\phi}_{1}=e^{A_{1}\left(t_{\Sigma}\right)}$ and $\hat{\phi}_{2}=e^{A_{2}\left(T-t_{\Sigma}\right)}$ and $S$ is the saltation matrix that relates the disturbance in the switching event as $\delta x_{\Sigma+}=S \delta x_{\Sigma-}$ (see Fig. 1(b)), which is computed as ${ }^{14}$

$$
S=I+\frac{\left(f_{+}\left(x\left(t_{\Sigma}\right), t_{\Sigma}\right)-f_{-}\left(x\left(t_{\Sigma}\right), t_{\Sigma}\right)\right) n^{T}}{n^{T} f_{-}\left(x\left(t_{\Sigma}\right), t_{\Sigma}\right)+\frac{\partial h\left(x\left(t_{\Sigma}\right), t_{\Sigma}\right)}{\partial t}},
$$

where $I$ is the identity matrix and $n$ is the normal vector to the switching surface. Using the monodromy matrix and the Floquet theory, it is possible to study the stability of periodic orbits in a PWL system. Particularly, considering the period-1 orbit, it will be stable if all the eigenvalues of the monodromy matrix are inside the unit circle, i.e. the maximum absolute value of eigenvalues is lower than 1 $(\mathrm{MVP}<1)$. In a general form, for a system with $n-1$ switching events per period, the monodromy matrix is computed as

$$
M_{m}=\hat{\phi}_{n} S_{n-1} \hat{\phi}_{n-1}, \ldots, \hat{\phi}_{2} S_{1} \hat{\phi}_{1} \text {. }
$$




\subsection{Matrix norm}

The matrix norm is an induced norm by the vector norm that maps the space of the matrices into $\mathcal{R}^{+} \cup\{0\}$, and it is defined by ${ }^{28,29}$

$$
\|A\|_{i}=\sup _{\forall x \neq 0} \frac{\|A x\|}{\|x\|}=\sup _{\|x\|=1}\|A x\|=\sup _{\|x\| \leq 1}\|A x\|,
$$

where the subscript $i$ indicates what vector norm is used. After some computations and using the norm $L^{2}$ (more commonly known as 2-norm or Euclidean norm), the matrix norm is expressed as $^{30}$

$$
\|A\|_{2}=\sqrt{\rho\left(A^{T} A\right)},
$$

where $\rho\left(A^{T} A\right)$ is the spectral radius, i.e. the maximum eigenvalue $\lambda$ that makes $A^{T} A-\lambda I$ singular, which is the norm that is used to analyze the behavior of the saltation matrices.

\section{Power Converters: Stability of the Period-1 Orbit}

In this section, the period-1 orbit of the current mode controlled boost and boostflyback converters are analyzed using the monodromy matrix. Fundamental theory about electronic devices, magnetically coupled inductors, circuits and power electronics can be found in Refs. 19 and 31.

\subsection{Boost converter}

The boost power converter analyzed in this paper is depicted in Fig. 2. In the circuit diagram $V_{\text {in }}$ is the input voltage and $V_{\text {ref }}$ is a scaled reference voltage. $L$ is the inductor, $C$ is the capacitor, $R$ is the load resistance. $D$ and $S_{D}$ resemble the diode

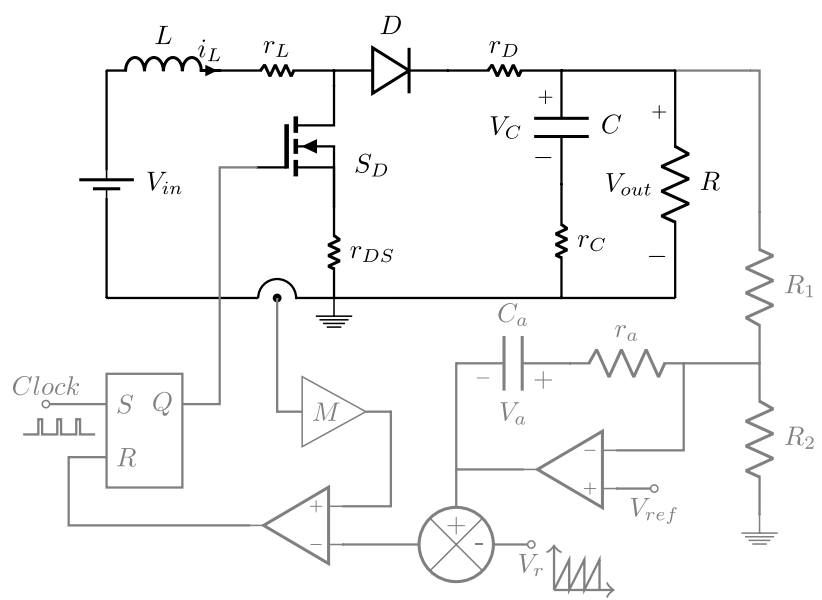

Fig. 2. Diagram of the boost power converter peak-current-mode-controlled. 
and the MOSFET. $r_{L}, r_{s}, r_{D}$ and $r_{C}$ are the internal resistances of the inductor, MOSFET, diode and capacitor, respectively. $R_{1}$ and $R_{2}$ constitute a voltage divider. As the converter is peak current-mode controlled, ${ }^{32} r_{a}$ and $C_{a}$ with the operational amplifier form the PI controller, and a compensation ramp is added to the output of the PI controller to obtain $i_{\text {ref }}$ and to avoid subharmonic behaviors. The inductor current $i_{L}$ multiplied by $M\left(M i_{L}\right)$ is compared with $i_{\text {ref }}$ to handle the flip-flop. For a complete description of the boost converter and the operation mode, the reader is referred to Refs. 20 and 33. The system has three state variables which are $X(t)=\left[\begin{array}{lll}i_{L} & V_{c} & V_{a}\end{array}\right]^{T}$, where $i_{L}$ is the inductor current, $V_{C}$ is the voltage in capacitor $C$ and $V_{a}$ is the voltage in capacitor $C_{a}$. The system can operate in three topologies depending on the switch and diode positions. In a compact form, the dynamical equations are written as

$$
\dot{X}= \begin{cases}A_{1} X(t)+B_{1} V_{\text {in }} S_{D}=1, & D=0 \\ A_{2} X(t)+B_{1} V_{\text {in }} S_{D}=0, & D=1 \\ A_{3} X(t)+B_{2} V_{\text {in }} S_{D}=0, & D=0 .\end{cases}
$$

The matrices $A_{1}, A_{2}$ and $A_{3}$ as well as vectors $B_{1}$ and $B_{2}$ are given by

$$
\begin{aligned}
& A_{1}=\left[\begin{array}{ccc}
-\frac{r_{L}+r_{S}}{L} & 0 & 0 \\
0 & -\frac{1}{T_{m}\left(1+k_{C}\right)} & 0 \\
0 & \frac{g}{T_{a}\left(1+k_{C}\right)} & 0
\end{array}\right], \quad B_{1}=\left[\begin{array}{c}
\frac{V_{\text {in }}}{L} \\
0 \\
-\frac{g k_{D} V_{\text {ref }}}{T_{a} V_{\text {in }}}
\end{array}\right] \text {, } \\
& A_{2}=\left[\begin{array}{ccc}
-\frac{r_{L}+r_{D}}{L}-\frac{r_{C}}{L\left(1+k_{C}\right)} & -\frac{1}{L\left(1+k_{C}\right)} & 0 \\
\frac{1}{C\left(1+k_{C}\right)} & -\frac{1}{T_{m}\left(1+k_{C}\right)} & 0 \\
\frac{g r_{C}}{T_{a}\left(1+k_{C}\right)} & \frac{g}{T_{a}\left(1+k_{C}\right)} & 0
\end{array}\right], \quad B_{1}=\left[\begin{array}{c}
0 \\
0 \\
-\frac{g k_{D} V_{\text {ref }}}{T_{a} V_{\text {in }}}
\end{array}\right] \text {, } \\
& A_{3}=\left[\begin{array}{ccc}
0 & 0 & 0 \\
0 & -\frac{1}{T_{m}\left(1+k_{C}\right)} & 0 \\
0 & \frac{g}{T_{a}\left(1+k_{C}\right)} & 0
\end{array}\right], \quad B_{2}=\left[\begin{array}{c}
0 \\
0 \\
-\frac{g k_{D} V_{\mathrm{ref}}}{T_{a} V_{\mathrm{in}}}
\end{array}\right]
\end{aligned}
$$

with $g=r_{a} / R_{1}, T_{m}=R C, T_{a}=r_{a} C_{a}, k_{c}=r_{c} / R$ and $k_{D}=\left(R_{1}+R_{2}\right) / R_{2}$. The voltage loop output $V_{k}$ is given by

$$
\mathrm{V}_{k}(t)=V_{\mathrm{ref}}\left(1+g k_{D}\right)-\left(\frac{g}{1+k_{C}}\right) V_{C}(t)-V_{a}(t),
$$

where $V_{\text {ref }}$ is the reference voltage. The reference current is

$$
i_{\text {ref }}=\mathrm{V}_{k}(t)-m_{a} \bmod \left(\frac{t}{T}\right)
$$


and the switching function is

$$
h_{B}(x(t), t)=k_{0}-M i_{L}-\frac{g}{1+k_{c}} V_{c}-V_{a}-m_{a} \bmod \left(\frac{t}{T}\right),
$$

where $V_{\text {ref }}$ is obtained from the desired output voltage $(20 \mathrm{~V})$ and the voltage divider formed by $R_{1}$ and $R_{2}$. In this way, $V_{\text {ref }}=20 R_{2} /\left(R_{1}+R_{2}\right)$ and $k_{0}=V_{\text {ref }}\left(1+g k_{D}\right)$. For all of the computations the parameter values $\operatorname{are}^{34}: L=165 \mu \mathrm{H}, C=150 \mu \mathrm{F}$, $r_{L}=0.04 \Omega, \quad r_{C}=0.03 \Omega, \quad r_{D}=0.01 \Omega, \quad r_{S}=0.055 \Omega, \quad T=60 \mu \mathrm{s}, \quad R_{1}=47 k \Omega$, $R_{2}=6.8 k \Omega, V_{\text {ref }}=2.5 \mathrm{~V}, r_{a}=4.7 k \Omega, C_{a}=2000 \mathrm{nF}, M=0.3$ and $m_{a}=0.445$. The monodromy matrix in continuous conduction mode is obtained as follows ${ }^{14}$ :

$$
M_{m}=\hat{\phi}\left(A_{2}, t=D T . . T\right) \cdot S_{1,2} \cdot \hat{\phi}\left(A_{1}, t=0 . . D T\right),
$$

where the time instant $t=D T$ corresponds to the time when the MOSFET switches from on (closed) to off (opened), as illustrated in Fig. 3. Figure 4 shows the evolution

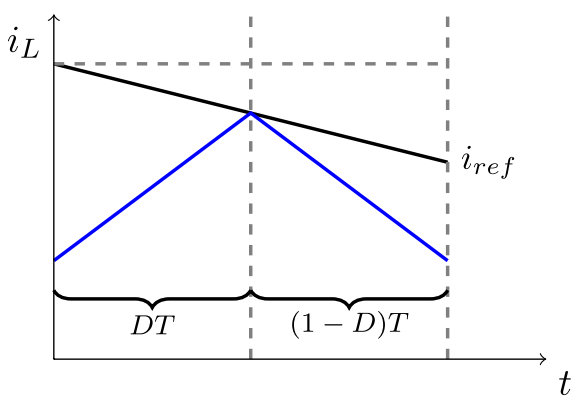

Fig. 3. Behavior of the inductor current $i_{L}$. The figure shows the instance when $i_{L}$ meets $i_{\text {ref }}$ and the switch changes the position.

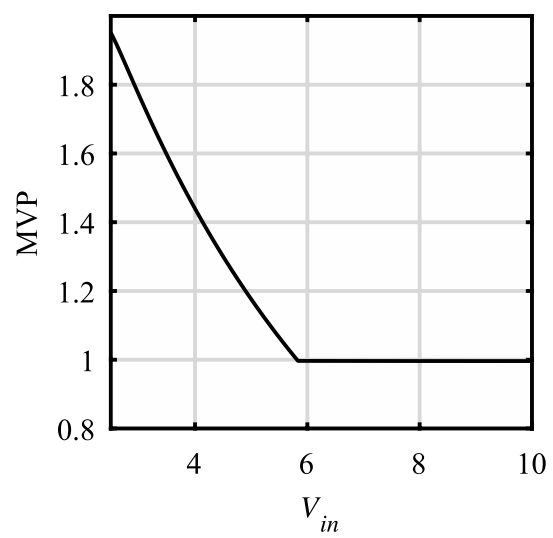

Fig. 4. Evolution of the MVP as $V_{\text {in }}$ varies (boost converter case). 
of the largest absolute value of the eigenvalues (MVP) of the monodromy matrix as the input voltage $V_{\text {in }}$ varies. For $V_{\text {in }}<5.84 \mathrm{~V}$, the period-1 orbit is unstable, and for $V_{\text {in }} \in(5.84 \mathrm{~V} 10 \mathrm{~V})$, it is stable.

\subsection{Boost-flyback converter}

This converter integrates two basic structures, as shown in Fig. 5. It has a pair of output capacitors $C_{1}$ and $C_{2}$, load resistance $R$, two diodes $D_{1}$ and $D_{2}$, and the MOSFET $S_{D}$. The main feature of this converter is that it has two coupled inductors, $L_{p}$ and $L_{s}$, with a coupled inductance $M_{L}$. The output of the converter is obtained as $V_{\text {out }}=V_{C_{1}}+V_{C_{2}}$; the parasitic resistances are $r_{p}, r_{s}, r_{D S}, r_{D_{1}}, r_{D_{2}}, r_{C_{1}}$ and $r_{C_{2}}$. Similarly to the boost converter case, the control parameters are $R_{1}, R_{2}, C_{a}$ and $r_{a}$, which define a PI external control loop. The state variables are $X=$ $\left[\begin{array}{lllll}i_{p} & i_{s} & V_{C_{1}} & V_{C_{2}} & V_{a}\end{array}\right]^{T}$, and they correspond to the primary and secondary inductor currents and the voltages on the capacitors $C_{1}, C_{2}$ and $C_{a}$, respectively. The system of equations that depend on the position of the switching devices are written as follows:

$$
\dot{X}= \begin{cases}A_{1} X+B_{1} V_{\text {in }} & \text { if } S_{D}=0, D_{1}=0, D_{2}=0, \\ A_{2} X+B_{2} V_{\text {in }} & \text { if } S_{D}=0, D_{1}=1, D_{2}=0, \\ A_{3} X+B_{3} V_{\text {in }} & \text { if } S_{D}=0, D_{1}=0, D_{2}=1, \\ A_{4} X+B_{4} V_{\text {in }} & \text { if } S_{D}=0, D_{1}=1, D_{2}=1, \\ A_{5} X+B_{5} V_{\text {in }} & \text { if } S_{D}=1, D_{1}=0, D_{2}=0, \\ A_{6} X+B_{6} V_{\text {in }} & \text { if } S_{D}=1, D_{1}=0, D_{2}=1 .\end{cases}
$$

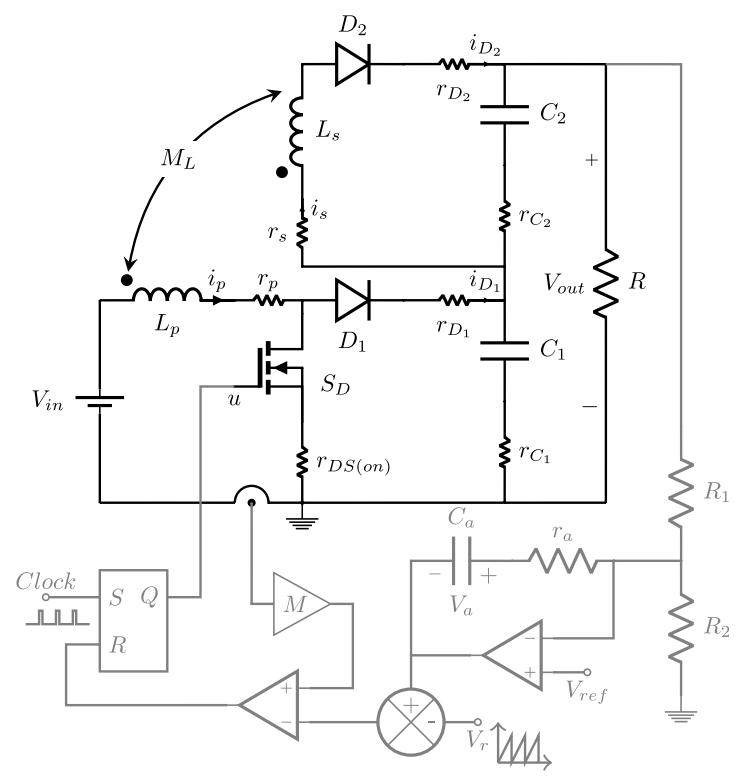

Fig. 5. Diagram of the boost-flyback converter peak-current-mode-controlled. 
However, when the system works in a particular period-1 solution, the converter evolves in four topologies in a period. At the beginning of period, while $S_{D}=1$ (i.e. the MOSFET is closed) the converter evolves through $\phi_{6}$ and $\phi_{5}$. After at $t=D T, S_{D}=0$ (the MOSFET opens) and the system evolves according to the dynamic defined by $\phi_{4}$ and $\phi_{3}$. In Appendix A, a brief summary related to obtaining these equations is presented. The matrices $A_{3}, B_{3}, A_{4}, B_{4}, A_{5}, B_{5}, A_{6}$ and $B_{6}$ are given by:

$$
A_{3}=\left[\begin{array}{ccrrr}
0 & 0 & 0 & 0 & 0 \\
0 & A_{3_{22}} & \frac{k_{a_{2}}}{L_{s}} & -\frac{k_{b_{2}}}{L_{s}} & 0 \\
0 & -\frac{k_{a_{2}}}{C_{1}} & -\frac{k_{c}}{T_{C_{1}}} & -\frac{k_{c}}{T_{C_{1}}} & 0 \\
0 & \frac{k_{b_{2}}}{C_{2}} & -\frac{k_{c}}{T_{C_{2}}} & -\frac{k_{c}}{T_{C_{2}}} & 0 \\
& & k_{C} g & k_{C} g & 0
\end{array}\right], \quad B_{3}=\left[\begin{array}{c}
0 \\
0 \\
0 \\
0 \\
-\frac{k_{D} g V_{\mathrm{ref}}}{T_{a} V_{\mathrm{in}}}
\end{array}\right],
$$

where $A_{3_{22}}=-\frac{r_{s}+r_{D_{2}}+r_{C_{2}} k_{b_{2}}}{L_{s}}, A_{3_{52}}=\frac{g\left(r_{C_{2}} k_{b_{2}}-r_{C_{1}} k_{a_{2}}\right)}{T_{a}}$.

$$
A_{4}=\left[\begin{array}{ccccc}
A_{4_{11}} & A_{4_{12}} & A_{4_{13}} & A_{4_{14}} & 0 \\
A_{4_{21}} & A_{4_{22}} & A_{4_{23}} & A_{4_{24}} & 0 \\
\frac{k_{a_{1}}}{C_{1}} & -\frac{k_{a_{2}}}{C_{1}} & -\frac{k_{c}}{T_{C_{1}}} & -\frac{k_{c}}{T_{C_{1}}} & 0 \\
-\frac{k_{b_{1}}}{C_{2}} & \frac{k_{b_{2}}}{C_{2}} & -\frac{k_{c}}{T_{C_{2}}} & -\frac{k_{c}}{T_{C_{2}}} & 0 \\
A_{4_{51}} & A_{4_{52}} & \frac{k_{c} g}{T_{a}} & \frac{k_{c} g}{T_{a}} & 0
\end{array}\right], \quad B_{4}=\left[\begin{array}{c}
\frac{L_{s}}{n} \\
-\frac{M_{L}}{n} \\
0 \\
0 \\
-\frac{k_{D} g V_{\mathrm{ref}}}{T_{a} V_{\mathrm{in}}}
\end{array}\right],
$$

where $\quad A_{4_{11}}=-\frac{L_{s}\left(r_{p}+r_{D_{1}}+r_{C_{1}} k_{a_{1}}\right)+r_{C_{2}} k_{b_{1}} M_{L}}{m}, \quad A_{4_{12}}=\frac{M_{L}\left(r_{s}+r_{D_{2}}+r_{C_{2}} k_{b_{2}}\right)+r_{C_{1}} k_{a_{2}} L_{s}}{m}$, $A_{4_{13}}=-\frac{k_{a_{1}} L_{s}+k_{a_{2}} M_{L}}{m}, \quad A_{4_{14}}=\frac{k_{b_{1}} L_{s}+k_{b_{2}} M_{L}}{m}, \quad A_{4_{21}}=\frac{M\left(r_{p}+r_{D_{1}}+r_{C_{1}} k_{a_{1}}\right)+r_{C_{2}} k_{b_{1}} L_{p}}{m}$, $A_{4_{22}}=-\frac{L_{p}\left(r_{s}+r_{D_{2}}+r_{C_{2}} k_{b_{2}}\right)+r_{C_{1}} k_{a_{2}} M_{L}}{m}, \quad A_{4_{23}}=\frac{k_{a_{1}} M_{L}+k_{a_{2}} L_{p}}{m}, \quad A_{4_{24}}=\frac{k_{b_{1}} M_{L}+k_{b_{2}} L_{p}}{m}$, $A_{4_{51}}=\frac{\left(r_{C_{1}} k_{a_{1}}-r_{C_{2}} k_{b_{1}}\right) g}{T_{a}}, A_{4_{52}}=\frac{\left(r_{C_{2}} k_{b_{2}}-r_{C_{1}} k_{a_{2}}\right) g}{T_{a}}$.

$$
A_{5}=\left[\begin{array}{ccccc}
-\frac{\left(r_{p}+r_{D S(\mathrm{on})}\right)}{L_{p}} & 0 & 0 & 0 & 0 \\
0 & 0 & 0 & 0 & 0 \\
0 & 0 & -\frac{k_{c}}{T_{C_{1}}} & -\frac{k_{c}}{T_{C_{1}}} & 0 \\
0 & 0 & -\frac{k_{c}}{T_{C_{2}}} & -\frac{k_{c}}{T_{C_{2}}} & 0 \\
0 & 0 & \frac{k_{c} g}{T_{a}} & \frac{k_{c} g}{T_{a}} & 0
\end{array}\right], \quad B_{5}=\left[\begin{array}{c}
\frac{1}{L_{p}} \\
0 \\
0 \\
0 \\
-\frac{k_{D} g V_{\text {ref }}}{T_{a} V_{\text {in }}}
\end{array}\right]
$$


J.-G. Muñoz, A. Pérez \& F. Angulo

$$
A_{6}=\left[\begin{array}{ccccc}
A_{6_{11}} & A_{6_{12}} & -\frac{k_{a_{2}} M_{L}}{n} & \frac{k_{b_{2}} M_{L}}{n} & 0 \\
A_{6_{21}} & A_{6_{22}} & \frac{k_{a_{2}} L_{p}}{n} & -\frac{k_{b_{2}} L_{p}}{n} & 0 \\
0 & -\frac{k_{a_{2}}}{C_{1}} & -\frac{k_{c}}{T_{C_{1}}} & -\frac{k_{c}}{T_{C_{1}}} & 0 \\
0 & \frac{k_{b_{2}}}{C_{2}} & -\frac{k_{c}}{T_{C_{2}}} & -\frac{k_{c}}{T_{C_{2}}} & 0 \\
0 & A_{6_{52}} & \frac{k_{C} g}{T_{a}} & \frac{k_{C} g}{T_{a}} & 0
\end{array}\right], \quad B_{6}=\left[\begin{array}{c}
\frac{L_{s}}{n} \\
-\frac{M_{L}}{n} \\
0 \\
0 \\
-\frac{k_{D} g V_{\text {ref }}}{T_{a} V_{\text {in }}}
\end{array}\right],
$$

where $\quad A_{6_{11}}=-\frac{L_{s}\left(r_{p}+r_{D S(\mathrm{on})}\right)}{m}, \quad A_{6_{12}}=\frac{M_{L}\left(r_{s}+r_{D_{2}}+r_{C_{2}} k_{b_{2}}\right)}{m}, \quad A_{6_{21}}=\frac{M_{L}\left(r_{p}+r_{D S(\mathrm{on})}\right)}{m}$, $A_{6_{22}}=-\frac{L_{p}\left(r_{s}+r_{D_{2}}+r_{C_{2}} k_{b_{2}}\right)}{m}, \quad A_{6_{52}}=\frac{g\left(r_{C_{2}} k_{b_{2}}-r_{C_{1}} k_{a_{2}}\right)}{T_{a}}$. The constant $n$ is defined as $n=L_{p} L_{s}-M^{2}$. The output of the outer voltage loop is

$$
V_{k}=\left(1+g k_{D}\right) V_{\text {ref }}-g k_{c}\left(V_{C_{1}}(t)+V_{C_{2}}(t)\right)-V_{a}(t) .
$$

The current reference is

$$
i_{\mathrm{ref}}=V_{k}-m_{a} \bmod \left(\frac{t}{T}\right),
$$

and the switching function is

$$
h_{\mathrm{BF}}(x(t), t)=k_{0}-M i_{p}-g k_{c}\left(V_{C_{1}}+V_{C_{2}}\right)-V_{a}-m_{a} \bmod \left(\frac{t}{T}\right),
$$

where $k_{0}=V_{\text {ref }}\left(1+g k_{D}\right)$. The monodromy matrix is obtained as follows ${ }^{35}$ :

$$
\begin{aligned}
M= & \hat{\phi}\left(A_{3}, t=t_{2}, \ldots, T\right) \cdot S_{3} \cdot \hat{\phi}\left(A_{4}, t=D T, \ldots, t_{2}\right) \\
& \cdot S_{2} \hat{\phi}\left(A_{5}, t=t_{1}, \ldots, D T\right) \cdot S_{1} \cdot \hat{\phi}\left(A_{6}, t=0, \ldots, t_{1}\right)
\end{aligned}
$$

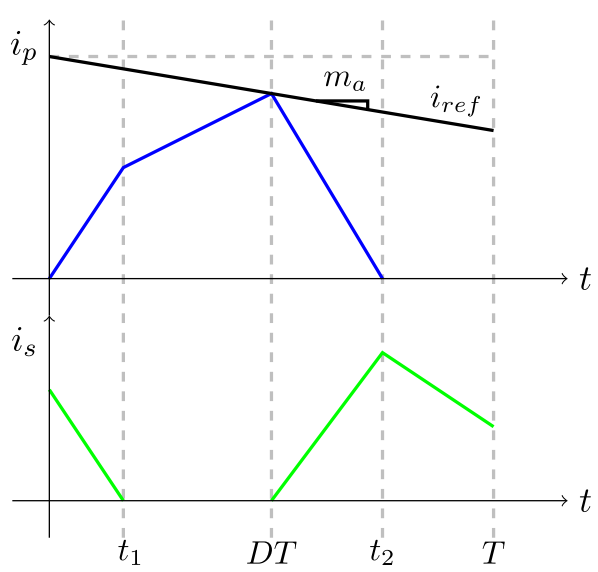

Fig. 6. Behavior of the inductor currents $i_{p}$ and $i_{s}$ (boost-flyback converter case). 


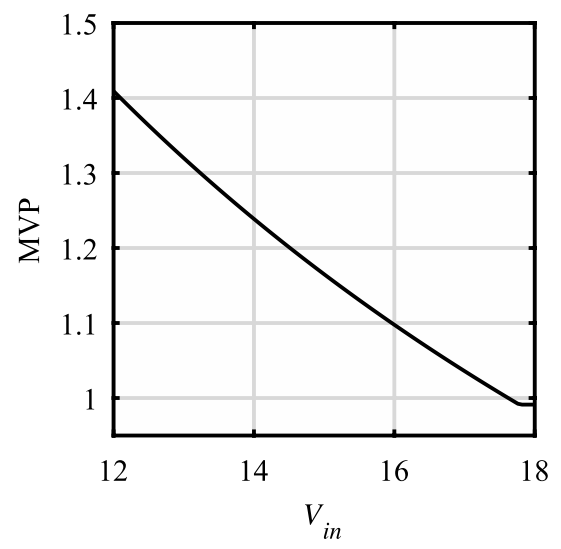

Fig. 7. Evolution of the MVP as $V_{\text {in }}$ varies (boost-flyback converter).

where the switching time instants are $t=t_{1}, t=D T$ and $t=t_{2}$, as they are shown in Fig. 6. The parameters used are $L_{p}=129.2 \mu \mathrm{H}, L_{s}=484.9 \mu \mathrm{H}, C_{1}=C_{2}=220 \mu \mathrm{F}$, $r_{p}=0.0368 \Omega, r_{s}=0.1307 \Omega, r_{D S}=0.04 \Omega, r_{C_{1}}=r_{C_{2}}=30 \mathrm{~m} \Omega, r_{D_{1}}=r_{D_{2}}=10 \mathrm{~m} \Omega$, $T=50 \mu \mathrm{S}, \quad V_{\text {ref }}=100 \mathrm{~V}, \quad R_{1}=1 M \Omega, \quad R_{2}=20 \mathrm{~K} \Omega, \quad r_{a}=390 \mathrm{~K} \Omega, \quad C_{a}=15 n \mathrm{~F}$, $M=0.196, m_{a}=0.392 / T$. Figure 7 shows the evolution of the MVP. The period-1 orbit loses stability near $17.7 \mathrm{~V}$.

\section{A New Method to Enhance the Stability of the Period-1 Orbit}

When the voltage gain increases above a limit value, it is necessary to add a compensation ramp. For a boost power converter, this limit is close to 2 ( $\left.V_{\text {out }}>2 V_{\text {in }}\right)$, and for the analyzed boost-flyback converter, it is close to 5.6 ( $\left.V_{\text {out }}>5.6 V_{\text {in }}\right)$. However, as the value of the slope increases, over-compensation occurs and deteriorates the response of the dynamical system. To guarantee the stability of the period-1 orbit as the voltage gain increases, it proposes changing the norm of the saltation matrix by re-tuning the parameters of the controller based on their original values. This approach affects the switching expression since the saltation matrix includes information from both the matrices and switching function. In the boost $(B)$ and the boost-flyback $(\mathrm{BF})$ converters, the switching functions $h_{(.)}(x(t))$ are given by:

$$
\begin{aligned}
& h_{B}(x(t), t)=k_{0}-M i_{L}-\frac{g}{1+k_{c}} V_{c}-V_{a}-m_{a} \bmod \left(\frac{t}{T}\right), \\
& h_{\mathrm{BF}}(x(t), t)=k_{0}-M i_{p}-g k_{c}\left(V_{C_{1}}+V_{C_{2}}\right)-V_{a}-m_{a} \bmod \left(\frac{t}{T}\right) .
\end{aligned}
$$

The switching function $h_{(.)}(x(t), t)$ affects the saltation matrix from both its time derivative and its normal vector $n$. As the slope compensation increases, the time derivative also increases and the norm of the saltation matrix decreases. This is the 
same reason behind adding a high period-small amplitude-function to the compensation ramp. The normal vector $n_{(.)}$for every converter is obtained as $n_{(.)}=\partial h / \partial X$.

$$
\begin{aligned}
& n_{B}=\frac{\partial h_{B}}{\partial X}=\left[\begin{array}{llll}
-M & -\frac{g}{1+k_{c}} & -1
\end{array}\right]^{T}, \\
& n_{\mathrm{BF}}=\frac{\partial h_{\mathrm{BF}}}{\partial X}=\left[\begin{array}{lllll}
-M & 0 & -g k_{c} & -g k_{c} & -1
\end{array}\right]^{T} .
\end{aligned}
$$

\subsection{Boost converter case}

The norm of the monodromy matrix of the boost converter as $V_{\text {in }}$ varies is depicted in Fig. 8(a). The norm close to where the system loses stability is approximately 7.3. Since the norm of the saltation matrix is similar to the norm of the monodromy matrix (Fig. 8(b)), the relation between the norms of the normal vector $\left(\left\|n_{B}\right\|\right)$ and the saltation matrix $(\|S\|)$ is studied.

The norm of the normal vector $n_{B}$ in terms of the parameters used in this paper is 1.0041, and the saltation matrix norm close to where the system loses stability is 7.3. Note that the norm of normal vector only depends on the controller parameters. Since the maximum value of the monodromy matrix was close to 14 , it proposes using an optimization algorithm such that the norm of the normal vector given by

$$
n_{B}=\left[\begin{array}{lll}
-M+\alpha & -\frac{g}{1+k_{c}}+\beta & -1+\gamma
\end{array}\right]^{T},
$$

be equal to $1.0041 / 2$, reducing the norm by $50 \%$. Remember that $M=0.3$ and $g /\left(1+k_{c}\right)=0.099$. The constants $\alpha, \beta$ and $\gamma$ must be determined through tuning. The sqp minimization method was implemented using the fmincon function provided by Matlab $^{\odot}$, and the values $\alpha=0.1570, \beta=0.0523$ and $\gamma=0.5233$ were

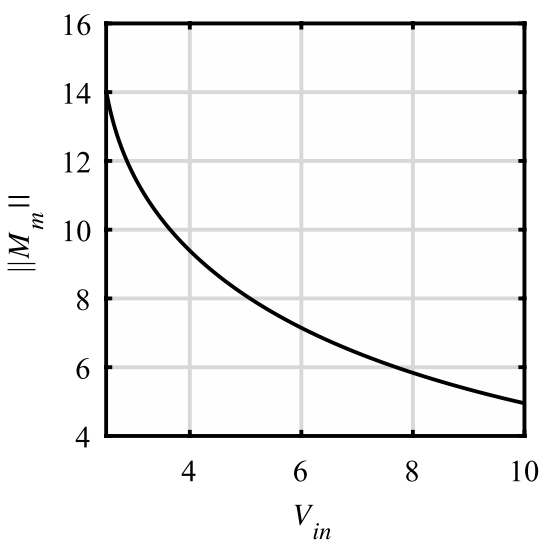

(a) $\left\|M_{m}\right\|$ versus $V_{\text {in }}$

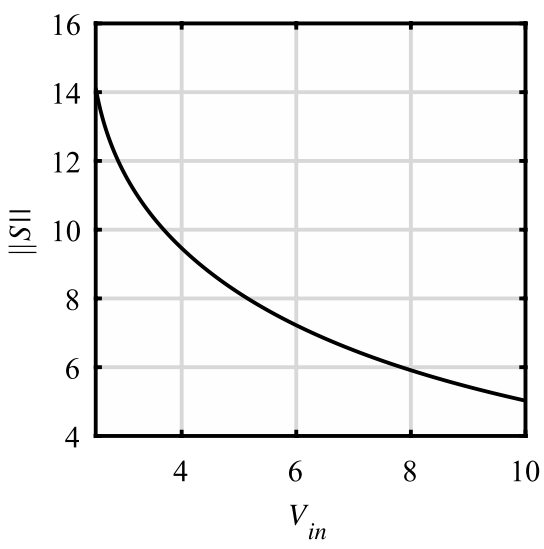

(b) $\|S\|$ versus $V_{\text {in }}$

Fig. 8. Matrix norms versus input voltage (boost converter case). 


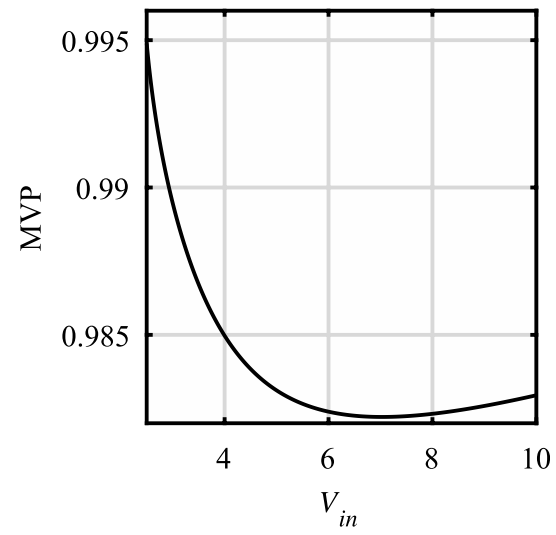

Fig. 9. Evolution of the MVP for the boost converter, as $V_{\text {in }}$ varies, after applying the new approach.

obtained. Using these values for the constants, the values of the controller were computed and determined to be $r_{a}=2.35 \mathrm{k} \Omega, C_{a}=4 \mu \mathrm{F}$ and $M=0.15$. The MVPA was again computed using these new values, and the result is depicted in Fig. 9. The figure shows that the period-1 orbit is stable for all of the ranges of the input voltage considered in the computations. Matlab R2017a was used and eight decimals were considered in all computations and routines.

\subsection{Boost-flyback converter case}

Since the system has three commutations, it is necessary to compute the matrix norm of these three matrices, which are shown in Figs. 10(a)-10(c). In Fig. 10(d), the behavior of the monodromy matrix norm as $V_{\text {in }}$ varies is displayed.

The saltation matrix that should be targeted is $S_{2}$ since it is the only matrix that was affected by varying $V_{\text {in }}$. Therefore, if the norm of $\left\|S_{2}\right\|$ is reduced, the spectral radius of $M_{m}$ will decrease and the stability range will be extended. The value of the monodromy matrix norm when the period-1 orbit loses stability $\left(V_{\text {in }}=17.7\right)$ is approximately 6.4 (see Figs. 7 and $10(\mathrm{~d})$ ) and the saltation matrix norm is approximately 35 for the same value of $V_{\text {in }}$. As the objective is for the system to work successfully for $V_{\text {in }} \in(12,18) \mathrm{V}$, the goal is obtained reducing the value of the norm from 55 to 34 , which implies a reduction of approximately $40 \%$. To do this, the value of the norm of the vector $\left\|n_{\mathrm{BF}}\right\|$ is reduced using the constants $\alpha, \beta$ and $\gamma$ as follows:

$$
n_{\mathrm{BF}}=\left[\begin{array}{lllll}
-M+\alpha & 0 & -g k_{c}+\beta & -g k_{c}+\beta & -1+\gamma
\end{array}\right]^{T} .
$$

Therefore, the vector norm $\left\|n_{\mathrm{BF}}\right\|$ was reduced by $45 \%$ to attain the stability of the period-1 orbit. As previously described, the minimization function fmincon was used to obtain the values of $\alpha=0.0761, \beta=0.2959$, and $\gamma=0.3884$. With these values, the parameters for the outer voltage and the current input gain $M$, were again computed. The new values for the elements were $r_{a}=100, \mathrm{k} \Omega, C_{a}=39 \mathrm{nF}$ and $M=0.12$. Figure 11 shows the evolution of the maximum absolute value of the 


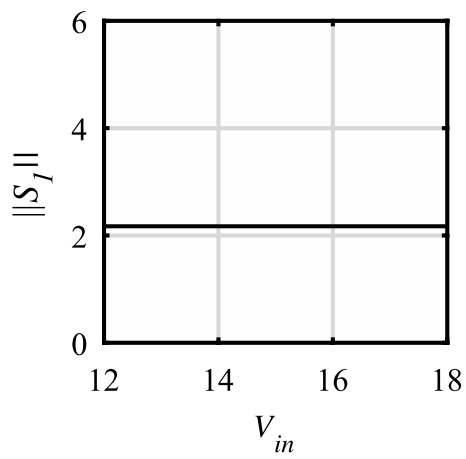

(a) $\left\|S_{1}\right\|$ versus $V_{\text {in }}$

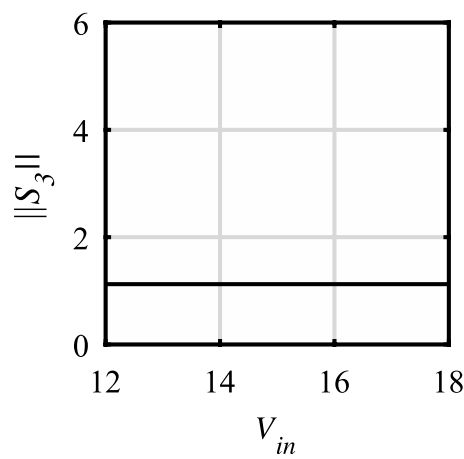

(c) $\left\|S_{3}\right\|$ versus $V_{\text {in }}$

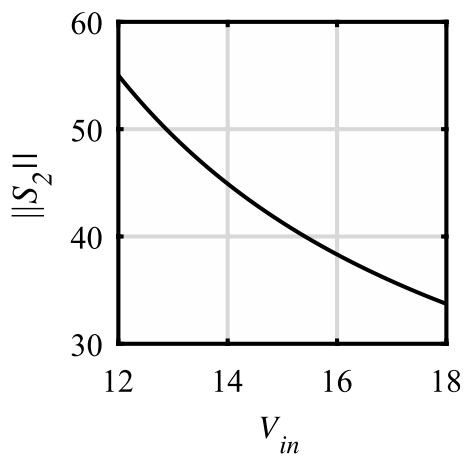

(b) $\left\|S_{2}\right\|$ versus $V_{\text {in }}$

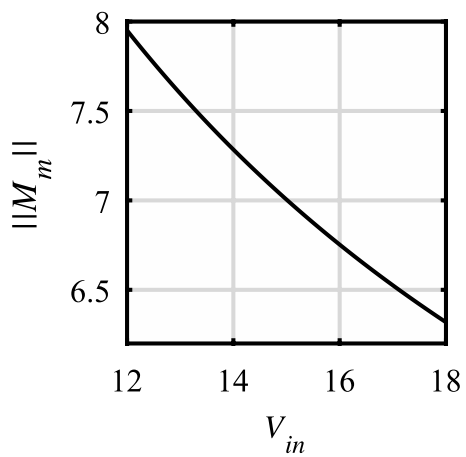

(d) $\left\|M_{m}\right\|$ versus $V_{\text {in }}$

Fig. 10. Matrix norms (boost-flyback converter case).

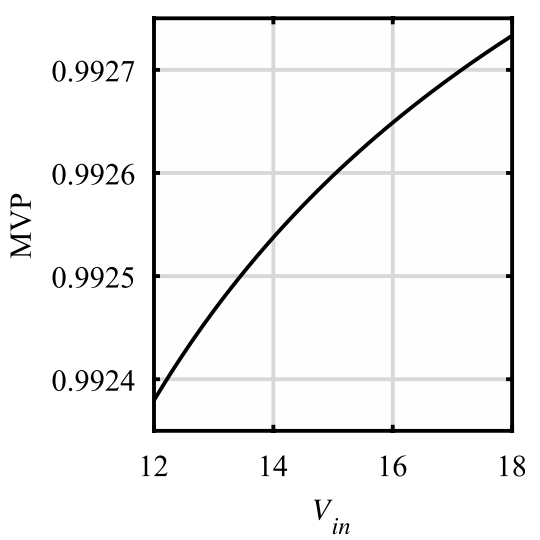

Fig. 11. Evolution of the MVP for the boost-flyback converter, as $V_{\text {in }}$ varies, after applying the new approach. 
eigenvalues as the input voltage varies. The period-1 orbit is now stable for the entire range of $V_{\text {in }}$.

\section{Experimental Results}

After obtaining successful results on a theoretical basis, the authors design and assemble an experimental set-up to prove the validity of the proposed controller. It is very complicated to control the boost-flyback converter since it is a 5-dimensional dynamical system, it has two diodes and one MOSFET, and its period-1 orbit evolves switching among four topologies. It is due to this complexity that this system was chosen to validate our approach. The results were obtained using two Schottky diodes C3D10060A ( $D_{1}$ and $\left.D_{2}\right)$. The controlled switch $(S)$ is a MOSFET IRFP260N with the driver TC4427. The values of the experimental parameters are the same as described in Sec. 3.2, resulting in a converter of 100 Watts of power. Figure 12 plots the peak current reference $\left(I_{c}=V_{k}-V_{r}\right)$, a ratio $M$ of the primary inductor current

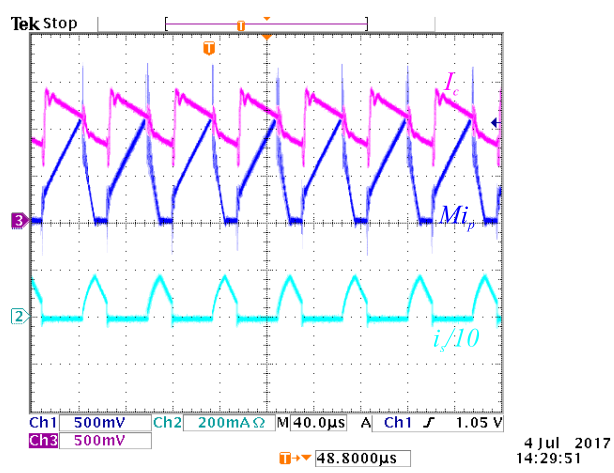

(a) Period-1 orbit for $V_{\text {in }}=18 \mathrm{~V}$

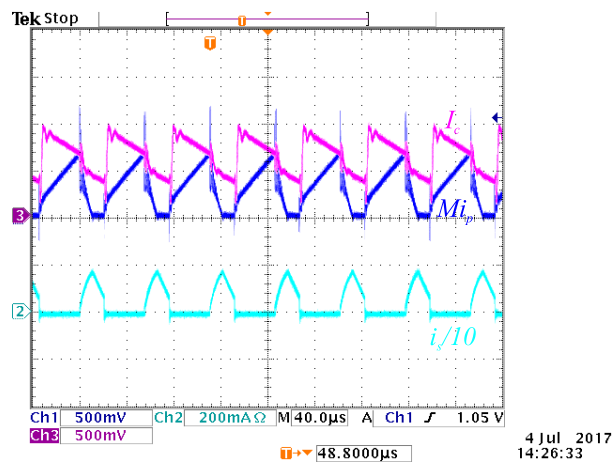

(c) Period-1 orbit for $V_{\text {in }}=18 \mathrm{~V}$ and using the proposed approach

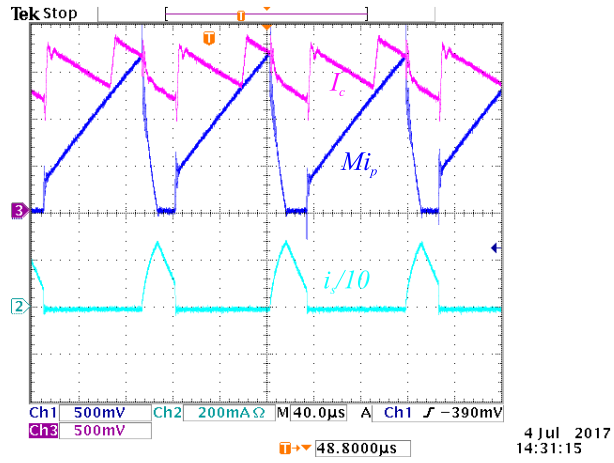

(b) Chaotic solution for $V_{\text {in }}=12 \mathrm{~V}$

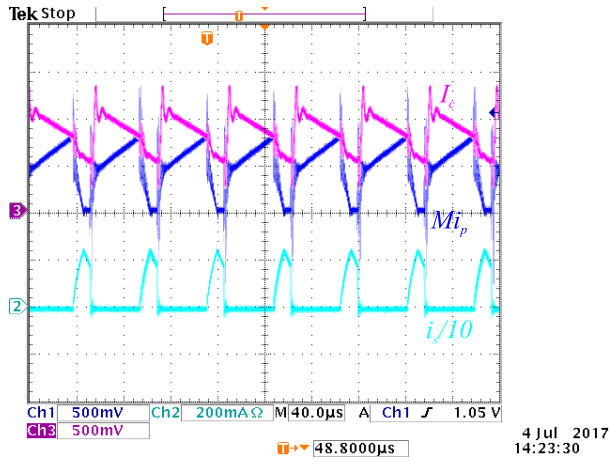

(d) Period-1 orbit for $V_{\text {in }}=12 \mathrm{~V}$ and using the proposed approach

Fig. 12. Boost-flyback converter experimental results. 
$\left(M i_{p}\right)$ and a ratio $(1 / 10)$ of the secondary inductor current $\left(i_{s} / 10\right)$. The experimental results were obtained using two values for the input voltage, $V_{\text {in }}=18 \mathrm{~V}$ and $V_{\text {in }}=12 \mathrm{~V}$. For $V_{\text {in }}=18 \mathrm{~V}$ and without using the tuning approach, the converter obtains a period-1 solution for $V_{\text {in }}=18 \mathrm{~V}$, as is shown in Fig. 12(a), but for $V_{\text {in }}=12 \mathrm{~V}$, the period-1 orbit loses stability (see Fig. 12(b)). In the second case, the proposed method is used and the controller is tuned according to the values computed after the reduction of the matrix norm. Figure 12(c) presents the results for $V_{\text {in }}=18 \mathrm{~V}$, which are stable. Figure $12(\mathrm{~d})$ presents that period-1 orbit remains stable for $V_{\text {in }}=12 \mathrm{~V}$ when the controller is tuned.

\section{Conclusions}

Control techniques for switched systems that are based on the analysis and change of the saltation matrix have emerged in recent years. Using this technique and information provided by the matrix norm, a new strategy for widening the stability range of the period-1 orbit was developed. This methodology first uses a set of values from the control parameters, and then, based on the matrix norm, it computes a new set of parameters so that the final controller is a better tuned version of the initial controller.

Although the application, computations and experiments were performed for power converters, the theory and methodology presented in this paper are broad enough that they could be applied to any other discontinuity system.

The specific advantages of this approach must be analyzed. For example, in the case of power converters, this approach is comparatively better than only increasing the slope value of the compensation ramp. Re-tuning the parameters using the matrix norm improves the performance of the system compared to only increasing the slope of the compensation ramp.

The tuning of the controller is carried out offline. The new controller is designed and implemented using the parameters $\alpha, \beta$ and $\gamma$ obtained after the tuning, and it remains unchanged.

It is important to note that the final tuning of the controller does not require much more effort than is currently used because analysis of the stability is already a forced task in control design. One only needs to compute the norms and minimize a function.

Finally, it is important to note that all results presented in this paper only include behavior analysis for linear loads.

\section{Acknowledgments}

This work was supported by Universidad Nacional de Colombia, projects 31492 and COLCIENCIAS under Contracts FP44842-052-2016 and National Ph.D. Programs 6172-2013, and Young Researchers Program 645-2014. 


\section{Appendix A. Equation System for the Boost-Flyback Converter}

In this section is briefly explained how the ODEs for the boost-flyback converter are found using Kirchhoff's law. First, the Kirchhoff's voltage law to compute $V_{p}$ and $V_{s}$ (voltage across the inductors $L_{p}$ and $L_{s}$ ) is applied. When $D_{2}=1, V_{p}$ and $V_{s}$ can be written as

$$
\begin{aligned}
& V_{p}=V_{\mathrm{in}}-V_{S_{D}}, \\
& V_{s}=-V_{D_{2}}-V_{C_{2}}-r_{C_{2}} C_{2} \frac{d V_{C_{2}}}{d t},
\end{aligned}
$$

where $V_{D_{2}}$ is the voltage across the diode $D_{2}$ and $V_{S_{D}}$ is the voltage across the MOSFET. $V_{S_{D}}$ depends on the switch position. For $S_{D}=1$, it can be expressed as $V_{S_{D}}=r_{D S_{\text {(on) }}} i_{p}$; when $S_{D}=0$, it can be expressed as $V_{S_{D}}=V_{D_{1}}+V_{C_{1}}+r_{C_{1}} C_{1} \frac{d V_{C_{1}}}{d t}$. After, using Kirchhoff's current law to compute $i_{D_{1}}$ and $i_{D_{2}}$ (currents flowing through diodes), it obtains

$$
\begin{aligned}
& i_{D_{1}}=C_{1} \frac{d V_{C_{1}}}{d t}+\frac{V_{C_{1}}}{R}+\frac{r_{C_{1}} C_{1}}{R} \frac{d V_{C_{1}}}{d t}+\frac{V_{C_{2}}}{R}+\frac{r_{C_{2}} C_{2}}{R} \frac{d V_{C_{2}}}{d t}, \\
& i_{D_{2}}=C_{2} \frac{d V_{C_{2}}}{d t}+\frac{V_{C_{1}}}{R}+\frac{r_{C_{1}} C_{1}}{R} \frac{d V_{C_{1}}}{d t}+\frac{V_{C_{2}}}{R}+\frac{r_{C_{2}} C_{2}}{R} \frac{d V_{C_{2}}}{d t} .
\end{aligned}
$$

From (A.3) and (A.4) and replacing $T_{C_{1}}=R C_{1}, T_{C_{2}}=R C_{2}, k_{a_{1}}=\left(1+r_{C_{2}} / R\right) /$ $\left(1+r_{C_{1}} / R+r_{C_{2}} / R\right), \quad k_{a_{2}}=\left(r_{C_{2}} / R\right) /\left(1+r_{C_{1}} / R+r_{C_{2}} / R\right), \quad k_{b_{1}}=\left(r_{C_{1}} / R\right) /\left(1+r_{C_{1}} /\right.$ $\left.R+r_{C_{2}} / R\right), \quad k_{b_{2}}=\left(1+r_{C_{1}} / R\right) /\left(1+r_{C_{1}} / R+r_{C_{2}} / R\right) \quad$ and $\quad k_{c}=(1) /\left(1+r_{C_{1}} / R+\right.$ $\left.r_{C_{2}} / R\right)$, it can obtain

$$
\begin{aligned}
& \frac{d V_{C_{1}}}{d t}=\frac{k_{a_{1}}}{C_{1}} i_{D_{1}}-\frac{k_{a_{2}}}{C_{1}} i_{D_{2}}-\frac{k_{c}}{T_{C_{1}}}\left(V_{C_{1}}+V_{C_{2}}\right), \\
& \frac{d V_{C_{2}}}{d t}=-\frac{k_{b_{1}}}{C_{2}} i_{D_{1}}+\frac{k_{b_{2}}}{C_{2}} i_{D_{2}}-\frac{k_{c}}{T_{C_{2}}}\left(V_{C_{1}}+V_{C_{2}}\right) .
\end{aligned}
$$

The state variable $V_{a}$ is found from operational amplifier as

$$
\frac{V_{\text {out }}}{R_{1}}-V_{\text {ref }} \frac{\left(R_{1}+R_{2}\right)}{R_{2}}=C_{a} \frac{d V_{a}}{d t} .
$$

Replacing $g=\frac{r_{a}}{R_{1}}, k_{D}=\frac{R_{1}+R_{2}}{R_{2}}$ and $T_{a}=r_{a} C_{a}$, it is obtained

$$
T_{a} \frac{d V_{a}}{d t}=g V_{\text {out }}-g k_{D} V_{\text {ref }}
$$

where $V_{\text {out }}$ is

$$
V_{\text {out }}=V_{C_{1}}+r_{C_{1}} C_{1} \frac{d V_{C_{1}}}{d t}+V_{C_{2}}+r_{C_{2}} C_{2} \frac{d V_{C_{2}}}{d t} .
$$

Finally, the equation system defined in (3.8)-(3.11) are found from (A.1), (A.2), (A.5), (A.6) and (A.8) and replacing the variables $V_{p}, V_{s}, V_{S_{D}}, V_{D_{2}}$ and $i_{D_{1}}$ depending 
on the switch and diodes positions, as follows:

- Equation (3.8) if $S_{D}=0, D_{1}=0, D_{2}=1$.

$$
\begin{aligned}
& * \frac{d i_{p}}{d t}=0 \\
& * V_{s}=r_{s} i_{s}+L_{s} \frac{d i_{s}}{d t} \\
& * V_{D_{2}}=r_{D_{2}} i_{D_{2}} \\
& * i_{D_{1}}=i_{p}=0 \text { and } i_{D_{2}}=i_{s}
\end{aligned}
$$

- Equation (3.9) if $S_{D}=0, D_{1}=1, D_{2}=1$.

$$
\begin{aligned}
& * V_{p}=r_{p} i_{p}+L_{p} \frac{d i_{p}}{d t}+M_{L} \frac{d i_{s}}{d t} \\
& * V_{s}=r_{s} i_{s}+L_{s} \frac{d i_{s}}{d t}+M_{L} \frac{d i_{p}}{d t} \\
& * V_{S_{D}}=r_{D_{1}} i_{D_{1}}+V_{C_{1}}+r_{C_{1}} C_{1} \frac{d V_{C_{1}}}{d t} \\
& * V_{D_{2}}=r_{D_{2}} i_{D_{2}} \\
& * i_{D_{1}}=i_{p} \text { and } i_{D_{2}}=i_{s}
\end{aligned}
$$

- Equation (3.10) if $S_{D}=1, D_{1}=0, D_{2}=0$.

* $V_{p}=r_{p} i_{p}+L_{p} \frac{d i_{p}}{d t}$

$* V_{S_{D}}=r_{D S \text { (on) }} i_{p}$

$* \frac{d i_{s}}{d t}=0, i_{D 1}=0$ and $i_{s}=i_{D_{2}}=0$

- Equation (3.11) if $S_{D}=1, D_{1}=0, D_{2}=1$.

* $V_{p}=r_{p} i_{p}+L_{p} \frac{d i_{p}}{d t}+M_{L} \frac{d i_{s}}{d t}$

* $V_{s}=r_{s} i_{s}+L_{s} \frac{d i_{s}}{d t}+M_{L} \frac{d i_{p}}{d t}$

$* V_{S_{D}}=r_{D S(\text { on) }} i_{p}$

* $V_{D_{2}}=r_{D_{2}} i_{D_{2}}$

* $i_{D 1}=0$ and $i_{s}=i_{D_{2}}$

\section{References}

1. D. He, S. Yu and L. Yu, Multi-objective nonlinear model predictive control through switching cost functions and its applications to chemical processes, Chin. J. Chem. Eng. 23(10) (2015) 1662-1669.

2. H. Cheng, C. Dong, W. Jiang, Q. Wang and Y. Hou, Non-fragile switched h $\infty$ control for morphing aircraft with asynchronous switching, Chin. J. Aeronaut. 30(3) (2017) $1127-1139$.

3. D. Xiang Fu, Y. Zhuang Liu, H. Guang Zhang, L. Xie and B. Li, The evolution of magnetization switching of lucro3 by the effect of mn doping, J. Alloys Comp. 735 (2018) 1052-1062.

4. M. C. F. Donkers, W. P. M. H. Heemels, N. van de Wouw and L. Hetel, Stability analysis of networked control systems using a switched linear systems approach, IEEE Trans. Autom. Control 56 (2011) 2101-2115.

5. M. Huang, P. Wen, Z. Zhang, B. Wang, W. Mao, J. Deng and H. Ni, Research on hybrid ratio of fuel cell hybrid vehicle based on advisor, Int. J. Hydrog. Energy 41(36) (2016) $16282-16286$. 
6. F. A. Silva, Clean energy microgrids [book news], IEEE Ind. Electron. Mag. 12 (2018) $79-80$.

7. S. Kouro, J. I. Leon, D. Vinnikov and L. G. Franquelo, Grid-connected photovoltaic systems: An overview of recent research and emerging pv converter technology, IEEE Ind. Electron. Mag. 9 (2015) 47-61.

8. B. Liu, M. Qiu, L. Jing, X. Wang and M. Chen, Design of high-performance bidirectional dc/dc converter applied for more electric aircraft, J. Eng. 2018(13) (2018) 520-523.

9. L. T. H. Jua, A. R. J. Juan, D. G. Aurelio and R. R. Juvenal, Eight levels multilevel voltage source inverter modulation technique, IEEE Latin Am. Trans. 16 (2018) 1121-1127.

10. M. Vidyasagar, Nonlinear Systems Analysis (Prentice Hall, 1993).

11. J.-J. E. Slotine and W. Li, Applied Nonlinear Control (Prentice Hall, Englewood Cliffs, NJ, 1991).

12. H. K. Khalil, Nonlinear Control (Pearson, New York, 2015).

13. A. Filippov, Differential Equations with Discontinuous Righthand Sides (American Mathematical Society Translations, 1978).

14. R. Leine, D. Van Campen and B. Van de Vrande, Bifurcations in nonlinear discontinuous systems, Nonlinear Dyn. 23(2) (2000) 105-164.

15. K. Tseng and T. Liang, Novel high-efficiency step-up converter, IEEE Proc. Electric Power Appl. 151 (2004) 182-190.

16. K. C. Tseng, J. T. Lin and C. A. Cheng, An integrated derived boost-flyback converter for fuel cell hybrid electric vehicles, in 2013 1st Int. Future Energy Electronics Conf. (IFEEC) Ainan, Taiwan, 2013, pp. 283-287.

17. S. M. Chen, C. Y. Wang and T. J. Liang, A novel sinusoidal boost-flyback ccm/dcm dc-dc converter, in 2014 IEEE Applied Power Electronics Conf. Exposition (APEC 2014) Fort Worth, TX, USA, 2014, pp. 3512-3516.

18. S. Amir, R. van der Zee and B. Nauta, An improved modeling and analysis technique for peak current-mode control-based boost converters, IEEE Trans. Power Electron. 30 (2015) 5309-5317.

19. R. W. Erickson and D. Maksimović, Fundamentals of Power Electronics (Springer, 2001).

20. T. M. Undeland, W. P. Robbins and N. Mohan, Power Electronics: Converters, Applications, and Design (John Wiley and Sons, New York, 2003).

21. D. Giaouris, S. Maity, S. Banerjee, V. Pickert and B. Zahawi, Application of filippov method for the analysis of subharmonic instability in dc-dc converters, Int. J. Circuit Theory Appl. 37(8) (2009) 899-919.

22. Y. Lai, S. Tan and S. Chan, Method of alleviating overcompensation problem in zerovoltage-transition pulse-width-modulation boost converters, Electron. Lett. 44 (2008) $444-445$.

23. A. Abusorrah, K. Mandal, D. Giaouris, A. E. Aroudi, M. M. Al-Hindawi, Y. Al-Turki and S. Banerjee, Avoiding instabilities in power electronic systems: Toward an on-chip implementation, IET Power Electron. 10(13) (2017) 1778-1787.

24. H. Asahara, S. Banerjee and T. Kousaka, Relationship of fast-scale and slow-scale instabilities in switching circuit with multiple inputs, Int. J. Circuit Theory Appl. 45(10) (2017) $1382-1391$.

25. A. E. Aroudi, A new approach for accurate prediction of subharmonic oscillation in switching regulators; part i: Mathematical derivations, IEEE Trans. Power Electron. 32 (2017) 5651-5665.

26. D. Giaouris, S. Banerjee, B. Zahawi and V. Pickert, Stability analysis of the continuousconduction-mode buck converter via filippov's method, IEEE Trans. Circuits Syst. I. Reg. Papers 55 (2008) 1084-1096. 
27. A. E. Aroudi, D. Giaouris, H. H. C. Iu and I. A. Hiskens, A review on stability analysis methods for switching mode power converters, IEEE J. Emerg. Sel. Top. Circuits Syst. 5 (2015) 302-315.

28. F. R. Gantmacher and J. L. Brenner, Applications of the Theory of Matrices (Courier Corporation, 2005).

29. P. Lancaster and M. Tismenetsky, The Theory of Matrices: With Applications (Elsevier, 1985).

30. C. D. Meyer, Matrix Analysis and Applied Linear Algebra (SIAM, 2000).

31. N. Mohan and T. M. Undeland, Power Electronics: Converters, Applications and Design (Wiley, 1995).

32. Y. Li, K. R. Vannorsdel, A. J. Zirger, M. Norris and D. Maksimovic, Current mode control for boost converters with constant power loads, IEEE Trans. Circuits Syst. I. Reg. Papers 59(1) (2012) 198-206.

33. Y. Chen, C. K. Tse, S. S. Qiu, L. Lindenmuller and W. Schwarz, Coexisting fast-scale and slow-scale instability in current-mode controlled dc/dc converters: Analysis, simulation and experimental results, IEEE Trans. Circuits Syst. I. Reg. Papers 55 (2008) 3335-3348.

34. D. Giaouris, S. Banerjee, O. Imrayed, K. Mandal, B. Zahawi and V. Pickert, Complex interaction between tori and onset of three-frequency quasi-periodicity in a current mode controlled boost converter, IEEE Trans. Circuits Syst. I. Reg. Papers 59 (2012) 207-214.

35. J.-G. Muñoz, G. Gallo, G. Osorio and F. Angulo, Performance analysis of a peak-current mode control with compensation ramp for a boost-flyback power converter, J. Control Sci. Eng. 2016 (2016) 1-14. 